\title{
Successful in vitro fertilization, twin pregnancy and labor in a woman with inherited propionic acidemia
}

\author{
Anna Wojtowicz ${ }^{1,2}$, Melanie Hill ${ }^{3}$, Strobel Spencer ${ }^{4}$, Godfrey Gillett ${ }^{5}$, Beata Kiec-Wilk ${ }^{1,6}$ \\ 'University Hospital, Cracow, Poland \\ ${ }^{2}$ Department of Obstetrics and Perinatology, Jagiellonian University Medical College, Cracow, Poland \\ ${ }^{3}$ Northern General Hospital, Dietetic Department Sheffield Teaching Hospital NHS, United Kingdom \\ ${ }^{4}$ Faculty of Medicine, Jagiellonian University Medical College, Cracow, Poland \\ ${ }^{5}$ Department of Clinical Chemistry, Sheffield Teaching Hospitals, Sheffield, United Kingdom \\ ${ }^{6}$ Department of Metabolic Diseases, Jagiellonian University Medical College, Cracow, Poland
}

Propionic acidemia (PA) is an inherited metabolic disorder, in which a defective form of the enzyme propionyl-coenzyme A carboxylase causes propionic acid accumulation. There are a few reports of singleton pregnancies that ended with successful births from mothers with PA. This is the first report of a twin pregnancy from in vitro fertilization (IVF) ending with successful delivery.

The presented 35-year old British Caucasian women had late-onset PA, was asymptomatic, and only on dietary treatment (protein approximately $0.8 \mathrm{~g} / \mathrm{kg}$ body mass/day, L-carnitine $1 \mathrm{~g} /$ day). After several unsuccessful induction of ovulation cycles and an artificial insemination, in vitro fertilization (IVF) was recommended. The patient was informed about the risk of metabolic decompensation. Two weeks after IVF, she developed a bacterial vaginal infection confirmed microbiologically. Nausea accompanying early onset of pregnancy resulted in reduced food intake, which induced symptoms of metabolic decompensation within a few days including: decreased concentration, disturbance in logical thinking, logorrhea, dizziness, tachypnea, tachycardia, and anxiety. Ultrasonography revealed ovarian hyperstimulation syndrome and monochorionic diamniotic twins. Laboratory tests found partial-compensated metabolic acidosis and electrolyte imbalance. Equalization of the metabolic disturbances in the patient was achieved according to current recommendations (i.e., $10 \%$ glucose, intravenous supplemental fluids and electrolytes, L-carnitine, ammonia scavengers, and a protein-free diet for 48 hours), in parallel with the application of cefuroxime. To provide the right amount of protein for ensuring proper development of a twin pregnancy, the patient received oral medical protein supplement XMTVI Maxamum (Nutricia). The pregnancy was also complicated by hypothyroidism and gestational diabetes mellitus controlled by intensive insulin treatment. Fetuses were monitored by serial ultrasound scans demonstrated $13 \%$ discrepancy of size between the fetuses (Fig. 1) and normal amniotic fluid volumes. At week 31, an upper respiratory tract infection occurred, followed by progressive symptoms of preterm delivery. The babies were delivered by cesarean section: the first twin weighed $1550 \mathrm{~g}$ (Apgar scores: 5/6/7), the second weighed $1340 \mathrm{~g}$ (Apgar scores: 7/8/9).

On the second day after delivery, the patient started vomiting and heart failure symptoms occurred as a result of exacerbation of PA. Laboratory tests showed anemia, electrolyte and metabolic disturbances. Normalization of patient's results was achieved with 2 units of red blood cells transfusion, continuous intravenous infusion of glucose with insulin controlled supplementation, fluids and electrolytes, as well as a high-sodium, high-carbohydrate, and protein-poor diet with supplementation of L-carnitine and low molecular weight heparin therapy. The control electrocardiography showed long QT syndrome, which was normalized by intravenous supplementation of potassium and magnesium salt.

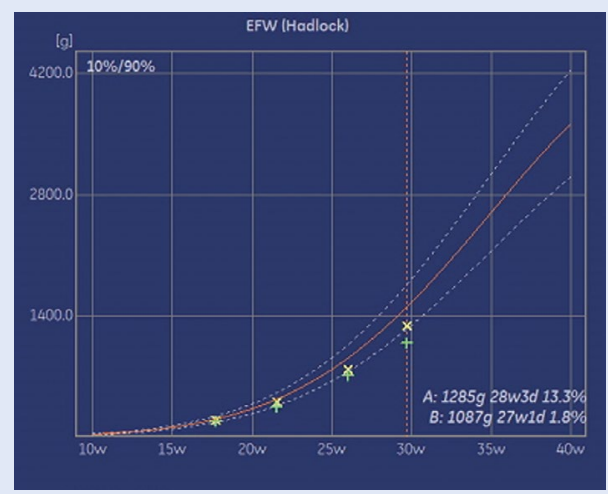

Figure 1. Fetal growth chart
Due to raised inflammatory parameters, the presence of Klebsiella pneumoniae in urine was revealed, which was treated with amoxiclav according to the antibiogram. Four weeks after delivery, all above regimens with dietary supplementation resulted in normalization of protein levels, with normal ammonia, lactate, and electrolytes levels.

This case report draws attention to the risk and the possibility of metabolic decompensation in PA patients during pregnancy. It is difficult to clearly indicate whether metabolic decompensation of PA patient was caused by IVF itself, or rather a twin gestation. In our opinion the IVF procedure, hormonal stimulation, induced decompensation in the initial period of pregnancy, while the metabolic decompensation just before the delivery was rather caused by the metabolic load of the body by twin pregnancy.

Corresponding author:

Anna Wojtowicz

Department of Obstetrics and Perinatology, University Hospital in Cracow, 23 Kopernika St, 31-501 Cracow, Poland

phone: +48600 243 156; e-mail: anna.3.wojtowicz@uj.edu.pl 
